\title{
Estudios de caso de la migración peruana "en Chile": un análisis crítico de las distorsiones de representación y representatividad en los recortes espaciales $^{1}$
}

\author{
Menara Lube Guizardi² y Alejandro Garcés ${ }^{3}$
}

\begin{abstract}
RESUMEN
El artículo aporta un análisis crítico de las investigaciones sobre migración peruana "en Chile" articulado alrededor de la representación y representatividad de los recortes espaciales que estos estudios operan. Como resultado, encontramos la reincidencia de una definición espacial "de lo peruano" que hace válida para todo el territorio nacional aquello que se estudió únicamente en la Región Metropolitana. Desarrollaremos nuestra discusión en tres partes: abordaremos datos y debates sobre los peruanos en Chile trabajados en la literatura científica disponible, señalando cómo se proyecta en ellos un juego de omisiones y emergencias que promueven una visibilización selectiva de las formas y consecuencias sociales de la migración peruana. A continuación, detallaremos los usos metodológicos que desde nuestro punto de vista contribuyen a resultados distorsionados acerca de la magnitud y/o generalización de las conclusiones de las investigaciones. Finalmente, debatiremos cómo estos estudios reinciden en ciertos conceptos nacionalistas hegemónicos sobre las relaciones de otredad en Chile.
\end{abstract}

Palabras clave: Migración peruana, colonialidad del saber, nacionalismo, identidad, recortes espaciales.

\begin{abstract}
The paper provides a critical analysis of research on Peruvian migration "in Chile" organized around the representation and representativeness of these studies in terms of spatial delimitation. We found that the recurrence of the spatial definition of "Peruvian", which represents the entire country, has only been studied in Santiago. We will develop our discussion into three parts: the presentation of data and debates on Peruvians in Chile in the available scientific literature, pointing out how these migrants are projected in a game of omissions and emergencies that promotes a selective visibility of forms and the social consequences of Peruvian migration. We will then detail the methodological applications that, from our point of view, contribute to distort results on the magnitude and/or generalization of these researches findings. Finally, we will discuss how these studies reiterate certain Chilean hegemonic nationalist concepts of otherness.
\end{abstract}

Key words: Peruvian migration, coloniality of knowledge, nationalism, identity, spatial delimitations.

\footnotetext{
1 Los autores agradecen a la Comisión de Ciencia y Tecnología de Chile (CONICYT) que financia este estudio a través de los proyectos: FONDECYT $\mathrm{N}^{\circ}$ 11121177 y No 11110246 . Artículo recibido el 14 de abril de 2012, aceptado el 6 de junio de 2013 y corregido el 26 de julio de 2013.
}

\footnotetext{
2 Universidad Alberto Hurtado (Chile) y Universidad de Tarapacá (Chile).

E-mail: menaraguizardi@yahoo.com.br

3 Instituto de Investigaciones Arqueológicas y Museo, Universidad Católica del Norte (Chile).

E-mail: ajgarces@gmail.com; agarces@ucn.cl
} 
Las migraciones internacionales vienen ocupando desde la década de 1990 un importante espacio en la agenda pública global, movilizando la generación y circulación de una serie de discursos articulados desde ámbitos académicos, desde la prensa, desde los Estados receptores y emisores de población migrante, y también desde los propios colectivos que protagonizan estos desplazamientos. La migración que tiene a Chile como país de destino -encabezada por migrantes provenientes de otros países de América Latina, pero en especial aquella que es realizada por migrantes originarios de territorios andinos- se ha convertido en un tema de discusión pública que motiva no solamente a investigadores de diversos campos de las ciencias sociales, sino también a representantes políticos que ven en la cuestión migratoria una circunstancia para la cual el país no está preparado, ni a niveles legales, ni a niveles sociales, y menos aún a niveles políticos. En este último sentido, la llegada de personas provenientes de Perú y Bolivia viene reavivando imaginarios y definiciones que traen a la luz las debilidades, incongruencias y asimetrías de la configuración territorial del proyecto chileno de identidad nacional. Peruanos y bolivianos, que durante mucho tiempo figuraron en el imaginario nacional hegemónico en Chile como los "otros" de la nación -como aquellos de quienes los chilenos se diferencian por sus supuestas atribuciones identitarias, étnicas, raciales y morales- son ahora parte del escenario urbano de Santiago, ocupando literalmente -por la adscripción geográfica al centro del país y por la construcción diaria de su presencia en el espacio público- aquello que el ideario nacional chileno define como "su centro".

Esta presencia peruana en Santiago provoca reacciones, mueve imaginarios y canaliza sentimientos colectivos de pertenencia nacional por parte de los chilenos, de los cuales no están libres los investigadores sociales. En gran medida, el discurso que hemos visto surgir en los trabajos científicos que dan cuenta de la presencia peruana "en Chile" reproducen estas categorías colectivas. Por un lado, desde un punto de vista académico, este fenómeno de reproducción de categorías político-sociales se hace notar en los usos discursivos repetidos por diferentes investigadores a la hora de definir espacialmente la presencia peruana en el país. Por otro, se hace notar también en la recurrencia de distorsiones metodológicas y analíticas. Estas distorsiones nos permiten observar que la manera como se denota el fenómeno viene demarcada por visiones de la realidad fuertemente ancladas en las categorías acerca de la centralidad de Santiago como epicentro del proyecto nacional, como centro de un principio de etnicidad e identidad que no opera de la misma manera en otras partes del país.

El presente artículo es resultado de un análisis de la bibliografía disponible acerca de la presencia de migrantes peruanos en Chile. Su objetivo central es apuntar el uso reiterado de una definición espacial "de lo peruano" que hace válido para todo el territorio nacional aquello que se estudió únicamente en la Región Metropolitana. En la misma medida, apuntamos distorsiones metodológicas que guardan una íntima relación con la ideología del centralismo de Santiago, y que se repiten de manera casi acrítica en muchos de los trabajos analizados. Nuestra principal indagación, en este sentido, se refiere a las estructuras e imaginarios de larga duración que operan condicionando este tipo de lectura "académica" acerca de la migración peruana. En última instancia, este interrogante nos lleva a plantear la cuestión de los colonialismos internos en la conformación de la relación centro-periferia en Chile, indagando en qué medida esto conlleva procesos de colonialidad del saber.

Nuestra discusión estará dividida en tres partes. En el apartado que sigue abordamos datos y debates sobre los peruanos en Chile encontrados en la literatura científica disponible sobre el tema. En esta sección usamos la referencia a los trabajos revisados para señalar cómo se proyecta en ellos un juego de omisiones y de emergencias que promueven una visibilización selectiva de las formas y consecuencias sociales de la migración peruana hacia Chile. A continuación, en el tercer apartado, pasamos a detallar los usos metodológicos -en especial referentes a la operación de los recortes espaciales de los estudios de caso- que desde nuestro punto de vista contribuyen a resultados distorsionados acerca de la magnitud y/o generalización de las conclusiones de las investigaciones. En el último apartado, aportamos nuestras conclu- 

DE LAS DISTORSIONES DE REPRESENTACIÓN Y REPRESENTATIVIDAD EN LOS RECORTES ESPACIALES

siones sobre la relación entre estos procesos de producción de discurso académico acerca de la otredad peruana en Chile, y la conformación de ideologías hegemónicas, y en cierto sentido modernas, referentes a la centralidad (nacional si se quiere) de Santiago.

\section{Emergencias y omisiones acerca de la "nueva migración peruana" en Chile}

Diferentes estudios coinciden en afirmar que Chile recibe una "nueva oleada migratoria" (Martínez, 2003: 1; Navarrete, 2007: 179; Schiappacasse, 2008: 23) peruana en la década de 1990 convirtiéndose en uno de los destinos prioritarios de la migración intrarregional sudamericana -un fenómeno que se habría intensificado especialmente a partir de 1995 (Araujo, Legua y Ossandón, 2002: 6; Godoy, 2007: 42; Núñez y Hoper, 2005: 291; Núñez y Torres, 2007: 7; Santander, 2006: 2; Stefoni, 2005a: 283-284)-. Este período coincide con el proceso de democratización vinculado al final de la dictadura de Pinochet, cuando Chile atraviesa una fase de estabilidad política y de importante crecimiento económico (Araujo et al., 2002: 8; Erazo, 2009: s/n; Jensen, 2009: 106; Martínez, 2005: 109; Poblete, 2006: 184). La aparición del país como un posible destino migratorio a niveles regionales es simultánea a la fuerte inestabilidad económica, política y social vivida por Perú, factor que actuó condicionando un flujo migratorio peruano hacia los países limítrofes (Arau et al., 2002: 9-10). En este contexto, Argentina apareció como un primer destino de la migración peruana en Latinoamérica, lo que ya era cierto para la migración boliviana desde hacía décadas. Esta realidad se mantuvo mientras la economía argentina sostuvo la paridad de cambio con el dólar, en el período anterior a la crisis del "corralito" (Lipszyc, 2004: 11), que determinó una parcial disminución de la migración intrarregional hacia el país. A la vez, tras los atentados del 11 de septiembre de 2001, las restricciones de fronteras impuestas por EE.UU. a los migrantes dificultaron sobremanera la entrada de latinoamericanos en Norteamérica (Araujo et al., 2002: 9). Si hasta 2001 Estados Unidos era el destino migratorio preferido de los peruanos, por ejemplo, a partir de esta fecha Chile y España surgen como nuevas posibi- lidades para el itinerario de estos migrantes andinos. Y es así que Chile pasa a figurar entre los países de destino de la migración peruana. A partir de fines de los 90 un especial interés sobre la presencia de estos migrantes en la sociedad chilena empieza a movilizar a los investigadores sociales (Cano y Soffia, 2009: 136), resultando en una serie de publicaciones sobre el tema que serían ya bastante expresivas en los primeros años del siglo XXI (Martínez, 2003: 1).

Sin embargo, determinar hasta qué punto y en qué términos esta es realmente una "nueva migración peruana" hacia Chile es una cuestión con un trasfondo político que nos acerca más a las ideologías territoriales, nacionalistas e identitarias chilenas, que a la migración peruana propiamente dicha. Como comenta Stefoni (2005a: 283-284), lo que hay de nuevo en esta migración no dice relación ni con el origen peruano de la población, ni con que el destino de este desplazamiento sea un territorio chileno. La "novedad" se refiere fundamentalmente a que estos migrantes lleguen por primera vez y de manera masiva a Santiago, ocupando un espacio que históricamente ha identificado a peruanos y bolivianos como los "otros" de una supuesta identidad blanca y "no indígena" chilena (Stefoni, 2005a: 266):

"La negación del origen indio como parte de la nación ha derivado no solo en la exclusión y discriminación de los pueblos originarios, sino en que la cultura dominante con un discurso homogeneizante intente barrer la diversidad de las culturas mapuches, aymaras y pehuenches, entre otras. En este sentido, la población indígena en Chile ha sido un 'otro' invisible, sistemáticamente silenciado en la formación de la nación y la identidad chilena. Representa todo aquello que no queremos ser y, por ende, es ocultado y negado. La inmigración andina nos vuelve a enfrentar con nuestra identidad mestiza y nos recuerda aquello que intentamos eliminar a fuerza de olvido. En otras palabras, nos enfrenta con nosotros mismos, ya que ies verdaderamente posible distinguir fenotípicamente un peruano de un chileno?" (Stefoni, 2005a: 266).

Aquí, la operación metonímica que identifica el "otro peruano" como un "indio" opera una clasificación política que es el 
reflejo de la dominación colonial: reflejo de cómo el colonialismo en cuanto estructura de producción de otredades y jerarquías de poder se actualiza a diario en la conformación de las ideologías de pertenencia nacional en Chile. Tal categoría, "indio", conjuga simultáneamente aspectos biológicos (raciales y racistas) y culturales, y es producto de la subordinación y negación de la humanidad de un grupo frente a otro que se construye como blanco, europeo (o euro-descendiente) y superior (Bello y Rangel, 2002: 40). En este sentido, la migración despierta y visibiliza los discursos hegemónicos del proyecto de construcción del Estado-Nación en Chile, como es la idea de que el país es una "excepcionali$\mathrm{dad}^{\prime \prime}$ en el contexto latinoamericano y que su supuesto "blanqueamiento" y "modernidad" le hacen contrastar con los vecinos Bolivia y Perú (Staab y Maber, 2006: 88-89), posicionándolo en un pretendido nivel superior. La globalización de la mano de obra operada por la migración internacional representaría un desafío especialmente complicado para naciones como la chilena, en la medida que

“Los sujetos inmigrantes -'Con' o 'sin' papeles- estarían en un espacio de sutura en ese Estado-Nación chileno que tiende a la exclusión (que ni siquiera ha dado el paso de reconocerse como un Estado intercultural, reconociendo a los pueblos originarios de su territorio). Chile se enfrenta, en tiempos de globalización económica, a salir de su propia estructura 'isleña' para plantearse situaciones no abordadas a nivel de estructuras institucionales como es el caso de la ciudadanía (...)" (Póo, 2009: 3).

Pero la separación ideológica con los vecinos andinos encuentra muchas evidencias empíricas en su contra. En gran medida, la migración en las regiones del norte de Chile, sobre todo donde las dos triples fronteras -la andina (Chile-Bolivia-Perú), y la Circumpuneña (Argentina-Chile-Bolivia) (González Miranda, 2009: 27) - constituye un fenómeno histórico de larga duración que invoca a procesos de movilidad social muy anteriores a la última década del siglo $\mathrm{XX}^{4}$ :

\footnotetext{
4 Estas afirmaciones contrastan con comentarios tejidos por autoras como Staab y Maber, quienes sin ninguna referencia bibliográfica sobre las migraciones
}

"Para el caso de las migraciones intrarregionales [sudamericanas hacia Chile], en especial de las migraciones fronterizas, los estudios señalan que estos movimientos poseen una profundidad histórica que se remonta incluso al periodo precolombino y que han sido parte de una historia compartida entre estos Estados nacionales fronterizos. Las migraciones fronterizas han supuesto continuidad y cambio en las zonas limítrofes, afectadas por distintos factores, como son los conflictos bélicos, la aparición de nuevas dinámicas económicas o la decadencia de estas, y la modificación de los límites territoriales, entre otros. (...) La presencia de extranjeros a nivel regional alcanzó una distribución desigual y altamente concentrada en algunos momentos de la historia, especialmente en las regiones del norte de Chile" (Tapia y Gavilán, 2006: 2).

En este mismo sentido, dando cuenta de una historicidad de la migración intrarregional hacia Chile, Cano y Soffia (2009: 135) afirman que en fines del siglo XIX el $67 \%$ de los extranjeros que vivían en el país eran latinoamericanos, siendo en su mayoría provenientes de Bolivia, Perú y Argentina. Las autoras citan como ejemplo de una continuidad histórica de la presencia de migrantes originarios de estos tres países, su llegada al Norte Grande chileno ocasionada por los ciclos productivos del salitre, en las primeras décadas del siglo XX. Sobre este tema González Pizarro (2008) afirmaría que:

"Durante los años que demarcan temporalmente el estudio en cuestión [1910 a 1930], Chile reflejó una situación paradójica en lo concerniente a la inmigración extranjera. Por un lado, observó una fuerte inmigración de los países vecinos en la frontera norte $y$, por otro, constató fuertes recelos de parte de la intelectualidad nacionalista por la llegada de oleadas de inmigrantes desde los países meridionales

históricas en Chile afirman que la migración peruana en este país es "a fairly recent phenomenon without strong historical precedent. Significant Peruvian migration into Chile did not occur until the early 1990s, after the liberal economic reforms of President Alberto Fujimori had begun to take effect in Peru and after the end of the Pinochet dictatorship in Chile" (Staab y Maber, 2006:91). 

DE LAS DISTORSIONES DE REPRESENTACIÓN Y REPRESENTATIVIDAD EN LOS RECORTES ESPACIALES

de Europa" (González Pizarro, 2008: 6162).

El comentario de González nos permite comprender la necesidad de considerar este fenómeno de acuerdo a sus especificidades espaciales e históricas y, no menos, de acuerdo a su relación con los discursos nacionalistas en boga para cada período. Lo que sí podemos considerar una constante, es la incidencia de estas ideologías de pertenencia nacional en Chile en el sentido de provocar en determinados momentos una sobreexposición de la presencia de ciertos grupos humanos, que en otros momentos y espacios del territorio nacional son más bien invisibilizados. Así, la visibilidad actualmente imputada a los peruanos como "ocupantes del centro de la nación chilena" dice relación con un proceso sui generis de demarcación de la otredad hacia este "otro migrante" que no se observa de esta misma manera en las fronteras al norte del país. Pero, en todos los casos, visibilidad e invisibilidad, emergencia y omisión, son dinámicas dicotómicas de carácter simbólico que se refieren a la construcción de fronteras nacionales y étnicas no necesariamente demarcadas por una línea euclidiana de separación de los territorios nacionales. Son más bien, parte de una construcción interna, de un capital cultural que se actualiza en la experiencia cotidiana. Son fronteras simbólicas que delimitan desde el habitus ${ }^{5}$ los otros internos y externos de la nación y que inciden sobre las prácticas sociales en el centro del país, subrayando la idea de oposición entre los chilenos y los "otros" andinos-indígenas.

Si por un lado es cierto que el último censo publicado (de 2002) presentó datos muy expresivos acerca de la inmigración internacional en Chile -visibilizando la presencia de 185 mil extranjeros residentes en el país,

\footnotetext{
5 Según Chauviré y Olivier (2008: 68), "Bourdieu presenta el habitus en términos de estructuras estructurantes (...) o de principio generador por analogías con las gramáticas generativas de Chomsky. El habitus es productor de acciones, al tiempo que es, a su vez, producido por el condicionamiento histórico social, lo que no significa totalmente determinado; engendra, de manera no mecánica, conductas objetivamente adaptadas a la lógica del campo social involucrado, y autorizadas, por tanto limitadas, por ella".
}

un número absoluto que supera el entonces máximo histórico de 135 mil extranjeros de fines del siglo XIX (Martínez, 2005: 111)también es cierto que en términos relativos, la presencia de extranjeros es más bien menor, apenas superando el 1\% del total de habitantes (Poblete, 2006: 1). De hecho, el total de inmigrantes en Chile sobre la población total fue más importante en otras épocas "como sucedió en la primera mitad del siglo $X X$, cuando se empinó por sobre un cuatro por ciento de la población total" (Martínez, 2005: 118). A raíz de estos datos históricos y estadísticos, el interés académico despertado por la presencia peruana en Santiago a fines del siglo $X X-y$ no menos su enunciación como un fenómeno "novedoso" "en Chile"nos lleva hacia las construcciones epistemológicas, metodológicas y discursivas sobre el "otro inmigrante" y acerca de los territorios "centrales" chilenos en los discursos académicos de las ciencias sociales. El presente artículo es una primera aportación hacia una crítica de estos discursos académicos, y tiene por finalidad deconstruir algunas categorías que, pese a carecer de respaldo empírico, son insistentemente reproducidas en los estudios acerca de la migración internacional en territorio chileno. En este sentido, concordamos con Martínez en su afirmación de que a raíz de la intensificación de la migración andina hacia la región central chilena,

\begin{abstract}
"Algunos sectores académicos difundieron la idea de que Chile se habría convertido en un 'polo' de atracción para inmigrantes, tanto de los países limítrofes como de fuera de América Latina, percepción que encontró mucho eco en los medios de comunicación. Estos se encargaron de crear la imagen de que en Chile se estaría produciendo una oleada migratoria donde las representaciones del inmigrante, muy variadas en realidad, no estuvieron desprovistas de estigmatizaciones, especialmente frente a los que provienen de Bolivia y Perú" (Martínez, 2003: 1).
\end{abstract}

En este aspecto, coincidimos con Segato (2007), asumiendo que la construcción de los discursos de rechazo y diferencia hacia peruanos y bolivianos en Chile, apuntan a que esta migración andina desestabiliza los paradigmas étnicos a partir de los cuales se construye el discurso hegemónico de la iden- 
tidad nacional chilena. En este sentido, consideramos que la experiencia migrante peruana constituye un locus privilegiado para estudiar los procesos de alteridad histórica entre Chile y Perú: no solamente porque representan un desafío al mismo orden euclidiano y moderno que dibuja los principio de límite de lo nacional-chileno; sino porque instaura un cierto grado de incertidumbre sobre aquello que se considera propio e impropio en el proyecto nacional -hegemonizado, por las élites nacionales y por su idea de homogeneidad tejida desde el centro (Santiago) y desplegada hacia las periferias del país-. Nos sumamos por lo tanto a Segato (2007) en su postura acerca de la necesidad de comprender los procesos históricos de construcción de las alteridades en el caso de cada nación para, a partir de este análisis enunciar el lugar dado en el discurso nacional a la diferencia cultural y a la asignación hegemónica de valores, principios, etnicidades o prácticas culturales "centrales" -en oposición a prácticas y gentes consideradas "refutables" o periféricas a la nación-:

"Este tipo de análisis política de la diferencia densa [en oposición a la frivolidad de las identidades globalizadas para el mercado] permitirá que se retire el énfasis de las fronteras culturales propicias al fraccionalismo y al congelamiento del proceso en su interior, y se lo coloque en el flujo interno de la historia propia de cada comunidad, en proceso constante de construcción. (...) Mi intento es el de una convocatoria a considerar la densidad de las diferencias culturales emergentes de antagonismos históricos complejos en cada nación y en cada región (...). O sea, se trata de una crítica a un mapa multicultural chato y esquemático, que diseña una diversidad fijada en el tiempo, reificada en sus contenidos y despojada de las dialécticas que le confieren historicidad, movilidad, y arraigo local, regional, nacional" (Segato, 2007: 20).

En relación a la invisibilización de estos procesos históricos de alteridad que generan la identidad nacional chilena, nos parece sorprendente que los títulos y apartados de artículos, libros, reseñas y ensayos que revisamos hablaran siempre de "migrantes peruanos en Chile", estando basados únicamente en estudios sobre el colectivo en Santiago. En el apartado que sigue, nos detendremos en un análisis metodológico de las incoherencias analíticas y empíricas de este uso discursivo.

\section{Transvaluación y focalización: las distorsiones metodológicas de los estudios sobre la migración peruana}

Al revisar detalladamente las investigaciones sobre los migrantes peruanos "en Chile" publicadas a partir de la década de 1990, nos encontramos con la reincidencia de un distorsionado proceso de generalización de los datos empíricos que nos pareció como mínimo curioso. Pese a que la casi totalidad de los estudios sobre el tema publicados entre 1990 y 2012 estén contextualizados en la Región Metropolitana, la mayor parte de los autores representa sus constataciones acerca del fenómeno en Santiago como si ellas fueran válidas para todo el territorio nacional. Esta representación de "todo Chile" a partir de experiencias sociales que tienen lugar, que se viven y que se observan en Santiago nos Ilamó la atención hacia dos fenómenos distintos pero ontológicamente interconectados. Por un lado, la ideología centralista chilena en su ímpetu de invisibilización de las diferencias, complejidades y diversidades regionales, que son subsumidas por la legitimidad de Santiago como "epicentro" del proyecto nacional'. Por otro, como decíamos anteriormente, la emergencia de unas representaciones de los migrantes peruanos como "los otros" de esta sociedad "central" chilena, que son reproducidas en los estudios sobre estos migrantes en Santiago aun cuando los autores expresan una postura crítica hacia esta ideología.

Estos dos fenómenos son elementos activos del proceso de construcción de una ideo-

\footnotetext{
6 Para un refinado análisis histórico del centralismo como ideología de las élites santiaguinas, como elemento estructurante del silenciamiento (económico, militar y político) de las demás regiones nacionales, y como principal motivación del control dictatorial/militar en la conformación del Estado chileno entre el siglo XIX y XX, véase Gabriel Salazar (2005).
} 
logía de "lo chileno" a partir de unos imaginarios modernos acerca de la constitución política de la relación centro-periferia, y se han convertido en puntos nodales para el estudio que actualmente llevamos a cabo sobre la migración transfronteriza en el Norte Grande de Chile -donde no encontramos la misma construcción de otredad de lo peruano que encontramos en los estudios de caso de Santiago, y donde las interacciones sociales con la capital se viven desde la incómoda perspectiva de aquellos que no se adaptan al centralismo del proyecto nacional santiaguino ${ }^{7}-$. En este sentido, es llamativo que las regiones de frontera -dada su conformación histórica, dado el intenso flujo de mercancías, dadas las particulares prácticas de movilidad de la población local- apenas figurasen en los estudios de caso de la migración peruana.

En gran medida, la bibliografía disponible sobre las migraciones internacionales en el norte de Chile está vinculada a los desplazamientos humanos ocurridos hasta la segunda mitad del siglo XX (Véase por ejemplo: Bähr, 1980; Gonzáles-Miranda, 2009; GonzálezPizarro, 2008, Larraín, 2000). Estos estudios dan cuenta de la historicidad de la presencia peruana y boliviana en territorios chilenos, apuntando hacia la existencia de estructuras sociales de larga duración que generan vinculaciones sui generis en estas áreas fronterizas. En muchos casos, sin embargo, hay una tendencia a asumir que estas dinámicas transfronterizas se inician y declinan en un periodo temporal determinado, lo que en gran medida invisibiliza la permanencia de circuitos de comunicación transnacional que resisten aun cuando los ciclos económicos que motivaron el flujo migratorio (la industria salitrera, por ejemplo) dejan de atraer manode-obra internacional hacia la zona.

\footnotetext{
7 Nuestra investigación aborda las condiciones laborales, relacionales, legales y simbólicas vividas por peruanos y bolivianos en las regiones de Arica-Parinacota, Tarapacá y Antofagasta, donde se establecen los límites nacionales-territoriales con Perú y con Bolivia. Estas tres regiones están compuestas por territorios que pertenecieron a Perú o a Bolivia en un momento anterior a las guerras entre estas naciones y Chile en el siglo XIX (Stefoni, 2005:266).
}

Por otro lado, también encontramos una variada bibliografía sobre la movilidad transfronteriza de los grupos étnicos entre territorios bolivianos, peruanos y chilenos (Véase: Imilan, 2007; Grebe, 1986; 2003; Gundermann y González, 2009; Gundermann y Vergara, 2009). Estos trabajos, pese a enfocar desplazamientos que son internacionales, se preocupan fundamentalmente con la dimensión étnica del fenómeno, dialogando muy poco con la bibliografía disponible sobre la realidad transnacional de los migrantes en territorios latinoamericanos. En este sentido, la migración peruana y boliviana en el norte de Chile fue estudiada antes como migración Aymara y Quechua, lo que en gran medida contribuye a la no visibilización de estos desplazamientos en su dimensión nacional, jurídica, política, y transnacional, si se quiere. Aún así, estos trabajos dan cuenta de una plasticidad y fluidez histórica de las fronteras con Perú y Bolivia, proporcionando los argumentos que ilustran cómo la realidad definidora de este "otro inmigrante" en el norte de Chile opera con sentidos y contenidos distintos de aquellos observados en la Región Metropolitana.

A partir de estas referencias es posible deducir que la invisibilización de los territorios de frontera entre Perú y Chile en los estudios de la presencia peruana en este segundo país es inversamente proporcional al énfasis dado a la capital como un locus protagonista, como escenario sine qua non de la experiencia migratoria en territorio chileno. Como afirmábamos anteriormente, esta preeminencia de la Región Metropolitana se materializa muy frecuentemente a partir de un mecanismo discursivo de generalización usado en una amplia gama de textos sobre el tema. Los estudios de caso reinciden en el uso de la expresión "en Chile" -"mujeres peruanas en Chile"; "explotación del trabajo peruano en Chile"; "dificultades documentales en Chile"aportando evidencias empíricas cualitativas (entrevistas, observaciones participantes, grupos focales) sobre la migración peruana realizados únicamente en Santiago.

En su conocido texto sobre el estado de la cuestión de los estudios migratorios, Cano y Soffia incurren en esta distorsión, al citar investigaciones realizadas en Santiago como "estudios de inmigrantes peruanos en Chile" 
(2009: 138). Este también es el caso para Godoy que hace frecuente uso de la expresión "mujeres peruanas en Chile" (2009: 45-46), citando las conclusiones y las entrevistas realizadas por autores que Ilevaron a cabo sus estudios cualitativos tan solamente en la Región Metropolitana. Cabib y Mora (2011) hablan de la ascendencia laboral de los peruanos "en Chile" estudiada a partir de 32 entrevistas de historia de vida. No hay ninguna mención sobre la región de residencia de los migrantes entrevistados. En el cuerpo del texto, sin embargo, todos los discursos de los peruanos citados hacen referencia a Santiago como locus de trabajo y vivienda: el lector tiende a entender que la totalidad de las entrevistas fueron realizadas en la Región Metropolitana. Sea este el caso o no, la omisión del recorte espacial y la ausencia de preocupación metodológica por una representación verdaderamente "nacional" -en que todas las regiones estén representadas- de la investigación nos dice de la operación del mismo proceso metonímico (el "en Santiago" como "en Chile") que encontramos en otros trabajos. Otros ejemplos de este uso metonímico de la expresión "en Chile" aparecen en los estudios de Araújo et al. (2002); Araya (2007); Cabib y Mora (2011); Godoy (2007); Jensen y Valdebenito (2010); Lipszyc (2004); Núñez y Torres (2007).

Stefoni es una de las pocas autoras que parece darse cuenta de esta limitación de las conclusiones obtenidas en los estudios de caso realizados solamente en Santiago (Stefoni, 2005a y 2005b). La autora afirma que:

“Es necesario señalar que este estudio está centrado en la comunidades de Santiago Centro, sin embargo ello no quiere decir que toda inmigración peruana pueda o deba reducirse a este tipo de experiencia. Sin duda que la inmigración proveniente del Perú cuenta con una larga historia en el país y obedece a distintos contextos políticos y económicos. El interés es dar cuenta de uno de los fenómenos asociados a la inmigración más reciente, pero quedan abiertas múltiples dimensiones de este fenómeno que podrán ser abiertos en otros trabajos" (Stefoni, 2005b: 321).

Esta precaución, no obstante, no impide que la autora haga uso de la expresión "migración peruana en Chile" en otros trabajos, en los que solamente aporta datos acerca de los peruanos en Santiago.

Los estudios que presentan datos referentes a otras regiones nacionales aportan por lo general, solo las estadísticas censales. Esto nos hace intuir una ausencia de interés por parte de los investigadores del tema en realizar trabajo de campo presencial y cualitativo en estas "regiones otras" del norte o sur chilenos. De los 75 textos recopilados (informes, capítulos de libro, artículos, tesis y ensayos) representativos de estudios de caso sobre la migración peruana en Chile en las últimas dos décadas, solamente 3 habían realizado trabajo de campo presencial afuera de la Región Metropolitana. Son estos: el trabajo de Méndez y Cárdenas (2012) sobre mujeres migrantes (peruanas, colombianas y bolivianas) en Antofagasta; el estudio de Erazo (2009) también realizado en esta última ciudad, pero centrado en las migraciones latinoamericanas ampliamente comprendidas, y el estudio de Tapia y Gavilán (2006) que aborda la realidad migratoria de la Región de Tarapacá para el período que va del siglo XIX al XXI. Por otro lado, también hay una serie de estudios de caso con trabajo de campo realizado en la Región Metropolitana que no inciden en generalizar la información extendiéndola hacia todo el territorio nacional. Estos trabajos asumen abiertamente la limitación geográfica de sus análisis, asumiendo el recorte espacial como "en Santiago". Véase por ejemplo: Luque (2004, 2005, 2007, 2009); Schiappacasse (2008); Staab y Maber (2006); Stefoni (2004). Nosotros también optamos por explicitar este recorte en algunos de nuestros trabajos (Garcés, 2007, 2011 y 2012). No obstante, en muchos de estos trabajos encontramos momentos en que los autores recurren a la expresión "en Chile" como marca generalizadora de las conclusiones de sus estudios.

Para mejor abordar este proceso de extensión nacional de lo que se estudia cualitativamente en el centro del país, tomemos como ejemplo el informe sobre migración peruana "en Chile" de Araujo, Legua y Ossandón (2002). El texto es considerado uno de los primeros escritos sobre el tema y presenta un panorama general del fenómeno en el país, basándose, por un lado, en datos estadísticos referentes a todo el territorio nacional $y$, por otro, en entrevistas realizadas a representan- 

DE LAS DISTORSIONES DE REPRESENTACIÓN Y REPRESENTATIVIDAD EN LOS RECORTES ESPACIALES

tes de ONG's de defensa de los derechos de los peruanos ubicadas en Santiago y a inmigrantes peruanas que viven y trabajan en esta misma ciudad. Así, gran parte de la representatividad cualitativa del informe se restringe a actores sociales cuya acción está circunscrita a la Región Metropolitana. Es probable que las malas condiciones de acceso a la salud, a la vivienda, a la educación y al trabajo por parte de las migrantes peruanas (Araujo, Legua y Ossadón, 2002: 36-45) -que las autoras identificaron a partir de las entrevistas cualitativas realizadas-, también se verifiquen en otras regiones del país. Pero la cuestión está en que se tome esta probabilidad como un hecho, asumiendo de manera naturaliza$d a^{8}$ la transposición de esta realidad a todo Chile. Hay, por lo tanto, una asunción de que la percepción de las ONG's sobre lo que pasa en Santiago y la experiencia de las migrantes en esta localidad puedan básica y directamente ser replicadas para todo el territorio nacional. Aquí, la distorsión metodológica se refiere al uso desigual de una metodología cuantitativa censal -representativa de todas las regiones nacionales-, puesta lado a lado a una metodología cualitativa espacialmente delimitada que no necesariamente debiera permitir el mismo tipo de afirmaciones genéricas tejidas a partir de los datos estadísticos nacionales.

La tendencia de asumir los recortes espaciales de manera metodológicamente irreflexiva se repite también en el trabajo de Lipszyc, pero siguiendo otro patrón de distorsión analítica. Como afirma la autora, su estudio comparativo centra "el enfoque en mujeres que han migrado en los últimos 25 años en cuatro países: Perú y Ecuador como países expulsores, y Argentina y Chile como receptores" (Lipszyc, 2004: 3). Sin embargo, no se explicita en ningún momento cuáles fueron los espacios elegidos para la investigación en Chile. Tampoco se expresa ninguna preocupación por los criterios de representatividad de estos espacios, dejando en abierto la cuestión metodológica de cómo se representa el territorio nacional en la muestra. Esta ausencia de preocupación reflexiva acerca de

\footnotetext{
8 Entendemos por naturalización el proceso de inscripción duradera de realidades sociales en el mundo natural (Bourdieu, 1999: 120).
}

esta generalización de las afirmaciones sobre la migración peruana en territorio chileno es como mínimo llamativa en fragmentos del texto en los que el uso de la expresión "en Chile" está respaldado por la referencia directa a bibliografías sobre el fenómeno en Santiago ${ }^{9}$. Una vez más, se asume como nacional, como "en Chile", los fenómenos estudiados básicamente en la Región Metropolitana, pero esta asunción ocurre ahora a partir de la omisión del recorte espacial de la investigación acompañado de un fuerte énfasis bibliográfico en estudios realizados en la capital chilena.

Un tercer patrón de generalización "nacional" a partir del uso acrítico de la expresión "en Chile" se encuentra en el trabajo de Núñez y Holper. Aquí, las autoras explicitan con precisión que su trabajo de campo estuvo enfocado en Santiago (2005: 293), lo que les permite explicitar la dimensión de "estudio de caso" que tiene la investigación. Hay una preocupación formal con la delimitación de los dos espacios centrales de la investigación ${ }^{10}$, pero esto no impide que extiendan las conclusiones obtenidas como si fueran representativas del total de la experiencia de las mujeres peruanas en Chile. Así, afirman por ejemplo que "las nanas peruanas en Chile consideran sus cuerpos delgados como indeseables y no sanos" (Núñez y Holper, 2005: 301, énfasis añadido), habiendo entrevistado tan solamente nanas peruanas que viven y trabajan en la Región Metropolitana. Este impulso de generalización nacional de los datos obtenidos en un estudio de caso en el que se aplicó un recorte local limitado, salta a la vista cuando en las conclusiones del texto las autoras afirman que: "en este artículo, hemos analizado dos espacios sociales diferentes y centrales en la vida cotidiana de las trabajadoras domésticas [peruanas] en Chile" (Núñez y Holper, 2005: 309, énfasis añadido). Aquí, nos queda definitivamente claro que se asume la experiencia de las peruanas

\footnotetext{
9 Véase Lipszyc, 2004: 22 - nota de pie de página número 26.

10 Estos espacios serían las casas de clase media donde las migrantes trabajan en Santiago, y las viviendas compartidas en el centro de la ciudad, donde ellas pasan su día libre en general los domingos de cada semana (Núñez y Holper, 2005:294).
} 
en la capital como representativa de lo que le ocurre al mismo colectivo migrante en todo el país.

Tijoux (2007) incurre en el mismo tipo de generalización realizado por Núñez y Holper al asumir como homogéneas y extensibles a todo Chile, las características conocidas de la migración femenina peruana en la capital. Pese a titular su estudio "Mujeres peruanas en Santiago" -haciendo patente la definición espacial del escenario en el que centra su estudio de caso- la autora también recurre a la expresión "en Chile" operando una y otra vez el mismo mecanismo generalizador:

“Las mujeres peruanas en Chile provienen principalmente de Trujillo, Chiclayo y Chimbote, tienen un nivel de escolaridad más alto que el promedio de las chilenas que trabajan en sus rublos, contando generalmente con educación básica, secundaria y en algunos casos universitaria" (Tijoux, 2007: 3).

En realidad, gran parte de la literatura sobre migración peruana en Santiago confirma estas mismas afirmaciones, pero en todos los casos estamos hablando de estudios realizados tan solamente en la capital. No sabemos si las mujeres peruanas que habitan la región norte del país provienen de Trujillo, Chiclayo y Chimbote: es altamente probable que vengan de otras regiones más al sur de Perú y más cercanas a la frontera con Chile. Tampoco sabemos si tienen el mismo nivel de educación de las mujeres que migran hacia Santiago, ya que apenas hay estudios sobre estas mujeres en el norte chileno. Esta asunción de que la migración peruana en Chile provendría prioritariamente del norte de Perú aparece en numerosos estudios, pero en ningún caso se cuestiona alguna variabilidad regional al respecto. Véase este mismo proceso de generalización en: Godoy (2004: 46), Santander (2006: 10). Poblete es un ejemplo interesante en este sentido, pues reproduce en su texto la idea de que los migrantes peruanos "en Chile" provienen mayoritariamente de Trujillo y Chimbote (2006: 205) y, páginas después, desglosa el dato citando que la información fue originalmente recopilada por Mujica (2004) en un estudio con peruanos realizado exclusivamente en la ciudad de Santiago (Poblete, 2006: 207).
Setién y Acosta (2011), a su vez, nos aportan un cuarto modelo de distorsión metodológica de los estudios de caso de la migración peruana. Las autoras se proponen a estudiar la "migración hacia Chile" (Setién y Acosta, 2011: 182), declarando desde un principio la intensión de operar un recorte que represente el fenómeno a niveles nacionales, lo que también observamos para el caso de Lipszyc (2004). Sin embargo, se explicita abiertamente que el recorte espacial de la investigación se restringe a Santiago, como también era el caso de Núñez y Holper y Tijoux. La diferencia está en que para Setién y Acosta la Región Metropolitana es efectivamente representativa de lo que ocurre a niveles nacionales con la migración peruana, de manera que consideran su recorte espacial justificado por una supuesta predominancia numérica de la migración internacional en la capital, "En el caso de Chile, las entrevistas se realizaron en totalidad en el Área Metropolitana de Santiago de Chile, dado que esta zona es la que concentra la mayoría de residencias de inmigrantes ecuatorianos y peruanos" (Setién y Acosta, 2011: 188).

Aquí, la opción metodológica está respaldada en asunciones que en partes carecen de validez empírica, y en partes carecen de validez analítica. En lo que dice relación al dato empírico, el que existan muchos más extranjeros peruanos en Santiago en números absolutos no significa que la migración peruana sea más relevante en esta región que en otras. Para mejor explicar esta afirmación, observemos los datos del último censo chileno (del 2002):

El Cuadro $N^{\circ} 1$ nos permite observar que dos de las cuatro regiones con más concentración absoluta de migrantes peruanos están en el Norte Grande chileno: Tarapacá y Antofagasta. Considerando además que el censo es anterior a la creación de la Región de Arica-Parinacota -originada en 2007 a partir de comunas anteriormente pertenecientes a Tarapacá- podemos inferir que las tres regiones del Norte Grande están entre las cuatro áreas con más concentración de migración peruana a niveles nacionales. No obstante, la diferencia en números absolutos entre las concentraciones migratorias de estas regiones y las de la Región Metropolitana es bastante expresiva. Sobre este punto, habría que considerar que Santiago es la región más pobla- 

DE LAS DISTORSIONES DE REPRESENTACIÓN Y REPRESENTATIVIDAD EN LOS RECORTES ESPACIALES

Cuadro $\mathrm{N}^{\circ} 1$

Inmigrantes peruanos para todas las regiones de Chile. Censo $2002^{11}$

\begin{tabular}{|l|r|}
\hline Región & Peruanos/as \\
\hline Metropolitana & 30.228 \\
Tarapacá & 4.912 \\
Antofagasta & 968 \\
Valparaíso & 1.171 \\
Coquimbo & 387 \\
Biobío & 351 \\
O'Higgins & 268 \\
Atacama & 211 \\
Maule & 205 \\
Los Lagos & 195 \\
Araucanía & 133 \\
Magallanes y Antártica & 30 \\
Aisén del Gen. Carlos Ibáñez del Campo & 25 \\
\hline
\end{tabular}

Fuente: INE, 2002.

da de Chile, y que en términos relativos la capital no necesariamente representa el área donde los migrantes peruanos son proporcionalmente más numerosos. Así, si analizamos las informaciones del censo 2002 sobre el número de migrantes peruanos en Tarapacá (4.912 personas) en relación al número total de habitantes de la región (428.594 personas), tenemos que estos migrantes representan un $1,15 \%$ de la población. Para el mismo año, lo peruanos significaban un $0,5 \%$ de la población santiaguina total. Este dato es importante porque desarticula el argumento acerca de la elección de Santiago que define este espacio como la región nacional donde la migración peruana se hace "excepcionalmente" presente y voluminosa. La estadística, nos ayuda a entender que el recorte geográfico del área de Santiago como locus sine qua non del estudio sobre migrantes peruanos en Chile se debe a lógicas de localización de otro orden: que no se vinculan necesariamente a la importancia numérica o proporcional del fenómeno. Así, la casi inexistencia de estudios sobre el fenómeno migratorio actualmente registrado en las regiones del norte, a ejemplo de lo que también pasa con la
Región de Valparaíso, dice relación antes con una distorsión de la mirada geográfica hacia el fenómeno, que con su supuesta inexistencia en estas regiones. Lo que está en juego es más bien el tipo de percepción acerca del fenómeno en la Región Metropolitana: la presencia peruana en la capital provoca un imaginario de "ocupación" del territorio nacional que los científicos sociales comparten con el total de la población y que condiciona el tipo de atención que dan al fenómeno en una u otra zona del país. En otras palabras, esta percepción resulta de estructuras de larga duración que reproducen la manera como en una u otra región se caracterizan las fronteras entre los unos y los otros del proyecto nacional chileno.

En lo que se refiere a la falta de validez analítica, la asunción de las autoras está basada en una equivocada comprensión acerca

\footnotetext{
11 Elaboración propia a partir de los datos del XVII Censo Nacional de Población y VI de Vivienda 2002. CELADE, División de Población de la CEPAL 2002-2006.
} 
de la representatividad espacial/poblacional en la investigación social. Desde un punto de vista cualitativo, el que determinada región concentre cuantitativamente a muchos migrantes no hace de ella representativa del total de regiones. Cualquier región puede representar o dar cobijo a relaciones sociales sui generis, únicas, particulares, que no se verifican en otros espacios locales. Así, la Región Metropolitana puede incluso ser indicativa de fenómenos que a lo mejor se verifican también en otras partes del país, pero en ningún caso ella será representativa de la totalidad de lo que ocurre en otras áreas. El que Santiago concentre un elevado número absoluto de migrantes no hace de la ciudad representativa de la complejidad de la experiencia migratoria "en Chile".

Este es un debate antiguo en las ciencias sociales y que dice relación, básicamente, con la diferencia de contenido y de representatividad que conforma la base epistemológica de las investigaciones cualitativas (consideradas aquí como "estudios de casos" situados en Santiago) por un lado, y de las investigaciones cuantitativas, por otro. Aquí, el argumento de que la investigación cualitativa realizada en Santiago tiene poder de representación del fenómeno "en Chile" porque los migrantes peruanos son numerosos en esta ciudad ignora que la lógica de la investigación cualitativa no permite generalizaciones de este tipo, puesto que su validez epistemológica está asentada en la noción de irreproductibilidad del caso estudiado: se hacen estudios cualitativos de un fenómeno para entender sus características sui generis. Pero, en segundo lugar, la especificidad de un fenómeno en un territorio dado no es ni metodológica ni epistémicamente definida por su importancia numérica. Aunque hubiera solamente un migrante peruano en Santiago, un estudio de caso sobre el tema podría ser relevante desde un punto de vista cualitativo. Una completa discusión sobre este tipo de equivocación, muy frecuente en las ciencias sociales, aparece en el clarificador texto de Mitchell (2006), "Case and Situation Analysis".

De manera general, todos los estudios que mencionamos anteriormente incurren en dos distorsiones del foco analítico que derivan de un no adecuado paso entre la fase empírica del trabajo y el análisis resultante de esta.
Por un lado, ellos operan una "transvaluación" [transvaluation], es decir, asumen que los elementos observados en el estudio de caso -que dependen marcadamente de un contexto específico, con su específico set de relaciones, movimientos y ubicaciones- son representativos de realidades, relaciones, territorios y fenómenos más amplios de lo que realmente pueden representar, definir o significar (Appadurai, 2000: 150). No se trata de una operación crítica de transición de contextos microsociales hacia macrosociales, como lo que se propone desde metodologías como el Extended Case Method (Burawoy, 1998:5). Es más bien un proceso en que se asume de manera acrítica y natural la representatividad de Santiago, y su legitimidad epistémica de "centro" que ilustra todo lo nacional, y que en gran medida tiene autoridad de significación sobre las demás regiones (periféricas) del país.

Al mismo tiempo, esta transvaluación implica un efecto inusitado de distorsión de los resultados obtenidos por estos estudios de caso. Paradójicamente, esta generalización compulsiva de lo que ocurre con la migración peruana en Santiago -la nacionalización metonímica de las conclusiones obtenidas en este contexto social determinado- termina provocando el proceso de progresivo encubrimiento, condensación y aglomeración de las particularidades contextuales del fenómeno estudiado, lo que le hace perder su riqueza particular (Appadurai, 2000: 150). En otras palabras, la contribución más pertinente de estos estudios -su capacidad de apuntar la configuración localizada, particular y única en Santiago de un fenómeno globalmente generalizado, observado en incontables ciudades del mundo, como lo es la migración internacional- es obscurecida por un mecanismo incoherente de generalización, incurriendo en una distorsión a la que Appadurai denominó "focalización" [focalization] (Appadurai, 2000: 150).

Este juego de generalización que nacionaliza como chileno lo que ocurre en el espacio central de la capital del país fue lo primero que nos hizo apuntar hacia una crítica de los textos leídos según criterios que en gran medida se proyectan fuera de las lógicas internas que guían sus exposiciones: según criterios que analizan no solamente lo que estos textos dicen, sino más bien lo que no dicen: las 
ausencias construidas, las naturalizaciones garantizadas por la omisión (Souza Santos, 2009: 100). La revisión bibliográfica nos permitió observar un interesante juego de visibilización e invisibilización de la presencia migratoria peruana en Chile operado por los estudios de caso acerca del fenómeno.

En gran medida, estos mecanismos de visibilización e invisibilización operan más allá de las intenciones críticas de los y las autoras, configurándose como una especie patrón repetido una y otra vez de manera casi inconsciente. La permanencia de las distorsiones analíticas provocadas por la superposición de estos mecanismos definidores de la mirada de los investigadores nos hizo indagar en qué medida estaríamos frente a una reproducción de categorías sociales y epistemologías de comprensión de la diferencia a las que también los investigadores están expuestos en cuanto miembros de una sociedad particular. Así, el reconocimiento de la reincidencia de recortes espaciales, nacionales, categóricos y discursivos en estos estudios de caso nos condujo a una reflexión sobre cómo esta reproducción casi acrítica de categorías de entendimiento, de visiones de mundo "autóctonas", se constituiría en cuanto habitus: en cuanto reproducción de estructuras estructuradas y estructurantes que dan los contornos de la experiencia de conformación de lo "nacional chileno". El debate sobre la manera como la migración peruana se representa en el imaginario científico de las ciencias sociales que estudian el tema nos permite, más allá de teorizar sobre la propia experiencia de los migrantes en Chile, discutir la construcción de imágenes dominantes acerca del espacio, de la otredad, de la retórica y de las categorías de comprensión que reactualizan en la producción de los saberes científicos un movimiento colonizador y moderno de definición de lo "nacional" en Chile.

\section{Reflexiones finales, más allá del discurso académico: etnicidad, centralismo y el principio moderno de colonialidad}

En las regiones del norte chileno, el "paradigma étnico" del centro de Chile -insepara- ble de la ideología racista del blanqueamiento de la población- no solamente no termina de tener cabida, como tampoco termina de construirse en cuanto sentido de sociabilidad. En parte porque la población no se reconoce homogéneamente en este paradigma, y en parte porque la identidad cultural de esta región ha sido histórica y predominantemente "andina", antes que "nacional chilena". En este sentido, el proceso identitario en el norte de Chile, los rasgos constitutivos de la manera como la gente se relaciona, se reconoce, se adscribe al mundo social, están dados antes por la vinculación a prácticas culturales exógenas a la ideología central del proyecto nacional chileno. Prácticas que como mencionaba Stefoni (2005a: 266), fueron silenciadas en el discurso homogeinizador del Estado, para permanecer vivas en la vida cotidiana de las gentes del Norte Grande.

Durante mucho tiempo, este conjunto cultural de prácticas "andinas" (ontológicamente vinculadas a las comunidades Aymaras, Quechuas y Atacameñas) constituyeron procesos de etnificación a niveles locales en el norte de Chile, haciéndose vivir como una subjetividad colectiva que las gentes dan por sentada y que se ubica en la intersección de la identidad con la cultura (Comaroff y Comaroff, 2009: 9). En gran medida, esta especie de "naturalización" del contenido predominantemente étnico de la identidad en el norte de Chile permitió su invisibilidad en los discursos políticos del centro de la nación. Paradójicamente, la invisibilización permitió la mantención de esta etnicidad, o por lo menos su no identificación por parte de las élites políticas nacionales. En tiempos de flujos globales en los que cada vez más turistas de todo el mundo viajan al norte de Chile para encontrar justamente una (por lo general descontextualizada) variedad de contenidos étnicos y/exotistas, esta etnicidad andina también viene adquiriendo características de empresa, y se encuentra "imbricada en la economía cotidiana como nunca lo estuvo antes" (Comaroff y Comarroff, 2009: 9). En localidades como San Pedro de Atacama, que dependen crecientemente del turismo internacional, este contenido de etnicidad andina local es no solamente deseado: es también subrayado, intensificado, transformado en hipérbole de "lo local". Este proceso es especialmente claro en las tiendas de "productos étnicos" 
dirigidas a los turistas internacionales de paso por San Pedro: todas ellas repletas de productos textiles venidos de Bolivia y vendidos como parte del "paisaje étnico" (Appadurai, 2000: 5) local del desierto chileno.

Este ejemplo nos permite enunciar una de nuestras principales conclusiones en el presente texto: la idea de que la visibilización de "lo peruano" en Santiago -su construcción como "el otro indígena"-, está profundamente vinculada con los juegos de emergencia y ocultamiento que el paradigma étnico nacional chileno plantea de manera desigual en diferentes espacios regionales de Chile. En este sentido, el discurso hegemónico de una composición poblacional "blanca" en el país ha sido puesto en práctica de manera asimétrica en las diferentes regiones, haciéndose fuertemente presente en el centro, a la vez que adquiriendo matices en la medida en que nos movemos del centro hacia las regiones más periféricas. Aquí, la centralidad de la ideología de pertenencia nacional se encuentra inscrita en su propia metáfora espacial, siendo la Región Metropolitana su espacio de más intensa materialidad.

Esta relación entre centro -como locus productor de una alteridad políticamente impuesta, homogeinizadora y dominante- y las periferias -como espacio invisibilizado, subordinado y adscrito a una eterna insuficiencia en relación al centro- tienen en sí un fuerte contenido "moderno", o más bien un contenido de cierta modernidad europea en su proceso de invención de la otredad contemporánea. Concordamos en este sentido con Enrique Dussel (1994) cuando para el caso de la modernidad europea afirma que, a contracorriente de su misma autodefinición normativa -como un movimiento histórico de racionalización de la experiencia social, de creciente dominio sobre el tiempo natural y sobre la propia naturaleza productora de dicha temporalidad; como un proceso de separación y quizás oposición a las cosmovisiones mágicas, encantadas, espirituales y "profanas" de los pueblos no cristianos; como un triunfo progresivo y total de la epistemología racional-cartesiana- es en realidad un proceso de invención mitológica. Invención de una mitología acerca de la conquista, de la subordinación, del derecho de explotación del otro. Nos interesa que esta mitología dice relación con la generación del principio de "centro" a partir del cual se diseñará una novedosa cosmovisión que posiciona Europa en el "eje gravitacional" del mundo, y que a la vez permite la creación de un principio de subjetividad, la europea, autojustificada por la concepción hegeliana de la "historia universal" como la única subjetividad posible. La subjetividad que autojustifica su derecho de subordinar a otros principios subjetivos. Así, Dussel asume que la "modernidad" coincide con la invención del principio de centro y con la invención del principio violento de universalización a partir de este centro, que es Europa; que son "los europeos".

En gran medida, la construcción de la categoría "centro", y la ideología de la primacía del centro como conductor de la normatividad del Estado-Nación en Chile recupera esta construcción de la modernidad europea reinscribiendo a niveles nacionales esta ideología de la conquista que el capitalismo hizo expandir inventando euclidianamente una "geografía internacional", que "naturaliza" la división del globo en naciones. Este también es un proceso que se observa en la construcción territorial de otros países latinoamericanos, pero en pocos de ellos se da la casualidad de que el "centro" en cuestión coincida con el "centro" espacial del territorio nacional, como ocurre en Chile ${ }^{12}$. Este tipo de reproducción de las lógicas modernas de construcción de la alteridad actúa potenciando la colonialidad de todo saber producido en las naciones donde esta práctica se mantiene. Esta colonialidad del conocimiento dice relación con la mantención histórica de invisibilidades: con el silenciamiento del otro, y con la negación del derecho de diferencia. Con la visibilización del centro y con su emergencia en cuanto productor de los discursos hegemónicos sobre pertenencia y exclusión.

En este sentido, el centralismo chileno es una ideología colonial de otredad, que cons-

\footnotetext{
12 Véase el ejemplo del caso brasileño en que la región hegemónica a niveles económicos, políticos y sociales es el sureste -ubicado en la costa atlántica involucrando ciudades como São Paulo y Río de Janeiro- y no la región central del territorio nacional. En una misma medida también podemos hablar del caso de Buenos Aires en Argentina.
} 

DE LAS DISTORSIONES DE REPRESENTACIÓN Y REPRESENTATIVIDAD EN LOS RECORTES ESPACIALES

truye a modo de "colonialismo interno" la justificación del dominio de un centro sobre sus supuestas periferias. Podemos considerar que esta reproducción de formas modernas de explotación y dominación actúa confirmando la "colonialidad del poder" (Maldonado-Torres, 2007: 129): el poder que se reinventa en un patrón colonialmente impuesto a modos de modernidad y, no menos, a modos de desarrollo (el desarrollo de Santiago, una Región Metropolitana, versus el subdesarrollo de las periferias, de las "provincias"). Ahora bien, como bien explicita Mignolo, esta colonialidad del poder se reproduce gracias a su confirmación en un nivel epistémico, es decir, su confirmación como estructura de conocimiento de la experiencia social, como "colonialidad del saber". Según Mignolo:

"La ciencia (conocimiento y sabiduría) no puede separarse del lenguaje; los lenguajes no son solo fenómenos 'culturales' en los que la gente encuentra su 'identidad'; estos son también el lugar donde el conocimiento está inscrito. Y si los lenguajes no son cosas que los seres humanos tienen, sino algo que estos son, la colonialidad del poder y del saber engendra, pues, la colonialidad del ser". (Mignolo, 2003: 669. En: Maldonado-Torres, 2007: 129).

De este modo, las prácticas discursivas que encontramos en el caso de los estudios sobre la migración peruana -que nacionalizan lo que es una realidad del "centro", y que invisibilizan las demás regiones chilenas en su diversidad de otredades posibles- deben ser tomadas en su contenido político último. Este contenido se refiere a que estas son prácticas discursivas que engendran, a través del lenguaje académico de las ciencias sociales, la colonialidad del poder y del saber producido acerca de las relaciones de otredad en Chile. La crítica a este discurso, creemos, se hace imprescindible si lo que pretendemos es producir conocimiento social no solamente sobre los otros, sino también sobre los "unos" en la producción de la espacialidad del poder.

\section{Referencias bibliográficas}

APPADURAI, A. Modernity at Large. Cultural Dimensions of Globalization. Minnea-
polis/London: University of Minnessota Press, 2000.

ARAÚJO, K.; LEGUA, M.C. y OSSANDÓN, L. Migrantes andinas en Chile. El caso de la migración peruana. Santiago de Chile: Fundación Instituto de la Mujer, 2002, p. $1-47$.

ARAYA MARTÍNEZ, M. Migrantes peruanos en el Chile contemporáneo. Cuaderno de Trabajo de la Universidad de Chile, 2007, No 4, p. 23-28.

BÄHR, J. Migraciones en el Norte Grande de Chile. Resultados de un análisis de movimientos migratorios entre los años 1965 y 1970. Revista de Geografía Norte Grande, 1980, No 7, p. 3-20.

BELLO, A. y RANGEL, M. La equidad y la exclusión de los pueblos indígenas y afrodescendientes en América Latina y el Caribe. Revista de la CEPAL, 2002, № 76, p. 39-54.

BOURDIEU, P. Efectos del lugar. En: BOURDIEU, P. La miseria del Mundo. Madrid: Akal, 1999, p. 119-124.

BURAWOY, M. The Extended Case Method. Sociological Theory, 1998, Vol. 16, $N^{0} 1$, p. 4-33.

BURAWOY, M. Introduction: Reaching for the Global. In: GOWAN, T. (editor). Global Etnography. Forces, Connections and Imaginnations in a Postmodern World. Berkeley-Los Angeles: University of California Press, 2000, p. 1-40.

CABIB, I. M. y MORA DEL VALLE, C. Capital Social e Inclusión Laboral. Una aproximación a las trayectorias de ascendencia laboral de migrantes Peruanos en Chile. Revista Polis, 2011, Vol. 10, No29, p. 147-163.

CANO, V. y SOFFIA, M. Los estudios sobre migración internacional en Chile: apuntes y comentarios para una agenda de investigación actualizada. Papeles de Población, 2009, No 61, p. 129-167.

CHAUVIRÉ, C. y FONTAINE, O. El vocabulario de Bourdieu. Buenos Aires: Editorial Atuel, 2008. 
CLIFFORD, J. Traveling Cultures. In: GROSSBERG, L. (editor). Cultural Studies. New York: Routdledge, 1992, p. 96-112.

COMAROFF, J. y COMAROFF, J. Etnicidad SA. Buenos Aires: Katz, 2009.

CSORDAS, T. Embodiment as a Paradigm for Anthropology. Ethos, 1990, Vol. 18, №1, p. 5-47.

DUSSEL, E. 1492. El encubrimiento del Otro. Hacia el origen del 'mito de la modernidad'. La Paz: Plural Editores, 1994.

ERAZO VEGA, C. Situación social de los inmigrantes latinoamericanos en la ciudad de Antofagasta, Chile. Hombre y Desierto. Una perspectiva Cultural, 2009, No 15, p. 139162.

GARCÉS H., A. Entre lugares y espacios desbordados. Formaciones urbanas de la migración peruana en Santiago de Chile. Santiago de Chile: Serie Documentos de la Escuela de Sociología - Universidad Central de Chile, 2007, No 2, p. 5-22.

GARCÉS H., A. De enclave a centralidad. Espacio urbano, comercio y migración peruana en Santiago de Chile. Gazeta de Antropología, 2011, Vol. 27, № 2, s/p.

GARCÉS H., A. Localizaciones para una espacialidad. Territorios de la migración peruana en Santiago de Chile. Revista Chungará, 2012, Vol. 44, No 1, p. 163-175.

GODOY, L. Fenómenos migratorios y Género: identidades 'remodeladas'. Psykhe, 2007, Vol. 16, No 1, p. 41-51.

GONZÁLEZ MIRANDA, S. El Norte Grande de Chile y sus dos triples fronteras: Andina (Perú, Bolivia y Chile) y Circumpuneña (Bolivia, Argentina y Chile). Cuadernos Interculturales, 2009, Vol. 7, No 13, p. 27-42.

GONZÁLEZ PIZARRO, J.A. La emigración boliviana en la precordillera de la región de Antofagasta, 1910-1930. Redes sociales y estudios de caso. Revista de Ciencias Sociales de la Universidad Arturo Prat, 2008, No 21, p. 61-85.
GREBE VICUÑA, M.E. Migración, identidad y cultura aymará: puntos de vista del actor. Revista Chungará, 1986, № 16-17, p. 205-223.

GREBE VICUÑA, M.E Procesos migratorios, identidad étnica y estrategias adaptativas en las culturas indígenas de Chile: una perspectiva preliminar. Santiago de Chile: Biblioteca Virtual Universal-Universidad de Chile, 2003.

GUNDERMANN KRÖLL, H. y GONZÁLEZ CORTÉS, H. Sociedades indígenas y conocimiento antropológico. Aymaras y Atacameños del siglo XIX al XX. Revista Chungará, 2009, Vol. 41, No 1, p. 113-164.

GUNDERMANN KRÖLL, H. y VERGARA, J.I. Comunidad, organización y complejidad social andinas en el norte de Chile. Estudios Atacameños: Arqueología y Antropología Surandinas, 2009, № 38, p. 107-126.

IMILAN, W. Socaireños en movimiento. Atacameños y Calama. Estudios Atacameños: Arqueología y Antropología Surandinas, 2007, No 33, p. 105-123.

JENSEN, M.F. Inmigrantes en Chile: la exclusión vista desde la política migratoria chilena. En: BOLOGNA, E. (organizador). Temáticas migratorias actuales en América Latina: remesas, políticas y emigración. Río de Janeiro: ALAP, 2009, p. 105-130.

JENSEN, M.F. y VALDEBENITO. De inclusiones y exclusiones: una perspectiva de la inmigración desde la Asociatividad en Chile. Miradas en Movimiento, 2011, № 3, p. 6-38.

LARRAÍN MIRA, P. Mujeres peruanas tras las huellas de los soldados. Revista de Historia, 2000, Vol. 33, p. 227-261.

LIPSZYC, C. Feminización de las Migraciones: Sueños y Realidades de las Mujeres migrantes en cuatro países de América Latina. Actas del Encuentro Caminar sin Miedos. Montevideo: Urbal, 2004, p.1-23.

LUQUE BRAZÁN, J.C. Transnacionalismo y enclave territorial étnico en la configuración de la ciudadanía de los migrantes perua- 

DE LAS DISTORSIONES DE REPRESENTACIÓN Y REPRESENTATIVIDAD EN LOS RECORTES ESPACIALES

nos en Santiago de Chile. Revista Enfoque, 2004, No 3, p. 81-102.

LUQUE BRAZÁN, J.C. Nacionalismo, Identidad Nacional y Sociedad Civil en la configuración de la ciudadanía de los migrantes peruanos en la ciudad de Santiago de Chile. Revista ICEA, 2005, Vol. 1, № 1, p. 129-148.

LUQUE BRAZÁN, J.C. Asociaciones políticas de inmigrantes peruanos y la 'Lima Chica' en Santiago de Chile. Migraciones Internacionales, 2007, Vol. 4, No 2, p. 121-150.

LUQUE BRAZÁN, J.C. La producción de la ciudadanía en la era de la migración: el caso de las asociaciones políticas de los peruanos en Santiago de Chile (1996-2006). Revista Ánfora, 2009, Vol. 16, № 27, p. 63-85.

MALDONADO-TORRES, N. Sobre la colonialidad del ser: contribuciones al desarroIlo de un concepto. En: CASTRO-GÓMEZ, S. y GROSFOGUEL, R. (editores.). El giro decolonial. Reflexiones para una diversidad epistémica más allá del capitalismo global. Bogotá: IESCO/Siglo del Hombre, 2007, p. 127-167.

MARCUS, G.E. On Ideologies of Reflexivity in Contemporary Efforts to Remake the Human Sciences. Poetics Today, 1994, Vol. $15, N^{\circ} 3$, p. 383-404.

MARCUS, G.E. Ethnography in/of the World System: The Emergence of Multi-Sited Ethnography. Annual Review of Anthropology, 1995, No 24, p. 95-117.

MARTÍNEZ PIZARRO, J. Breve examen de la inmigración en Chile según los datos generales del Censo de 2002. Documentos para Trabajo OIM, 2003, No 3, p. 1-20.

MARTÍNEZ PIZARRO, J. Magnitud y dinámica de la inmigración en Chile, según censo 2002. Papeles de Población, 2005, № 44, p.109-147.

MÉNDEZ CARO, L. y CÁRDENAS CASTRO, M. Hacia una construcción de un modelo comprensivo de análisis de la 'situación de migración' de mujeres sudamericanas en Chile. Psicoperspectivas: Individuo y Sociedad, 2012, Vol. 11, No 1, p. 252-272.
MITCHELL, J. C. Case and Situation Analysis. In: EVENS, T.M.S \& HANDELMAN, D. The Manchester School. Practice and Ethnographic Praxis in Anthropology. Manchester: Berghan Books, 2006, p. 13-22.

MUJICA, J. El desafío de la solidaridad: condiciones de vida y trabajo de los migrantes peruanos en Chile. Lima: OIT, 2004.

NAVARRETE YÁÑEZ, B. La quinta oleada migratoria de peruanos a Chile: los residentes legales. Revista Enfoques, 2007, N 7, p. 173195.

NÚÑEZ, L. y HOLPER, D. En el Perú, nadie se muere de hambre': pérdida de peso y prácticas de alimentación entre trabajadoras domésticas peruanas. En: BERG, U. y PAERREGAARD, K. (editores). El quinto suyo. Transnacionalidad y formación diaspórica en la migración peruana. Lima: Instituto de Estudios Peruanos, 2005, p. 291-313.

NÚÑEZ, N.R. y TORRES, C.E. Mujeres migrantes peruanas y salud reproductiva. Usuarias de consultorios de salud de la Zona Norte de la Región Metropolitana. Santiago de Chile: Fundación Instituto de la Mujer, 2007.

PASSARO, J. You Can't Take the Subway to the Field!: 'Village' Epistemologies in the Global Village. In: AKHIL, G. \& FERGU SON, J. (editors). Anthropological Locations. Boundaries and Grounds of a Field Science. Berkeley-Los Angeles: University of California Press, 1997, p. 147-162.

POBLETE MELIS, R. Educación intercultural: teorías, políticas y prácticas. La migración peruana en el Chile de hoy. Nuevos escenarios y desafíos para la integración. Tesis doctoral. Barcelona: Departamento de Antropología Social y Cultural-Universidad Autónoma de Barcelona, 2006.

PÓO, X.F. Imaginarios sobre migración peruana en la prensa escrita chilena: una mirada a la instalación de la agenda de la diferencia. Revista Faro, 2009, Vol. 5, No 9, p. 1-9.

SALAZAR, G. Construcción de Estado en Chile (1800-1837). Democracia de los "pueblos". Militarismo ciudadano. Golpismo 
Oligárquico. Santiago de Chile: Editorial Sudamericana, 2005.

SANTANDER, C.U. La migración peruana en el contexto del patrón de las corrientes migratorias en Chile: pasado, presente y futuro. Puerto Rico: Lasa Congress, 2006.

SCHIAPPACASSE CAMBIASO, P. Segregación espacial y nichos étnicos de los migrantes internacionales en el Área Metropolitana de Santiago. Revista de Geografía Norte Grande, 2008, № 39, p. 21-38.

SEGATO, R. L. La nación y sus otros: Raza, etnicidad y diversidad religiosa en tiempos de Políticas de la Identidad. Buenos Aires: Prometeo, 2007.

SETIÉN, M. L. y ACOSTA, E. Cuidados y flujos migratorios feminizados surnorte $y$ sur-sur: Negación de derechos y ciudadanía limitada. Revista Latina de Sociología, 2001, № 1, p. 182-208.

SOUZA SANTOS, B. Una epistemología del Sur. Buenos Aires: CLACSO Ediciones y Siglo XXI, 2009.

STAAB, S. \& MABER, K.H. The Dual Discourse about Peruvian Domestic Workers in Santiago de Chile: Class, Race, and a Nationalist Project. Latin American Politics and Society, 2006, Vol. 48, № 1, p. 87-116.
STEFONI, C. Inmigrantes Transnacionales. La formación de comunidades y la transformación en ciudadanos. En: BERG, U. y PAERREGAARD, K. (editores). El quinto suyo. Transnacionalidad y formación diaspórica en la migración peruana. Lima: Instituto de Estudios Peruanos, 2005a, p. 261-289.

STEFONI, C. Inmigración y ciudadanía. La formación de comunidades peruanas en Santiago y la emergencia de nuevos ciudadanos. Política, 2005b, № 43, p. 319-336.

TAPIA LADINO, M. y GAVILÁN VEGA, V. Diagnóstico de las migraciones fronterizas de la I Región de Tarapacá, Chile. Iquique: Universidad Arturo Prat, 2006.

TIJOUX, M.E. Peruanas migrantes en Santiago. Un arte cotidiano de la lucha por la vida. Polis - Revista de la Universidad Bolivariana, 2007, $\mathrm{N}^{\circ} 18, \mathrm{~s} / \mathrm{p}$.

TSING, A.L. Friction. An Ethnography of Global Connection. Princeton: Princeton University Press, 2005.

WESTON, K. The Virtual Anthropologist. In: AKHIL, G. \& FERGUSON, J. (editors). Anthropological Locations. Boundaries and Grounds of a Field Science. Berkeley-Los Angeles: University of California Press, 1997, p. 163-184. 